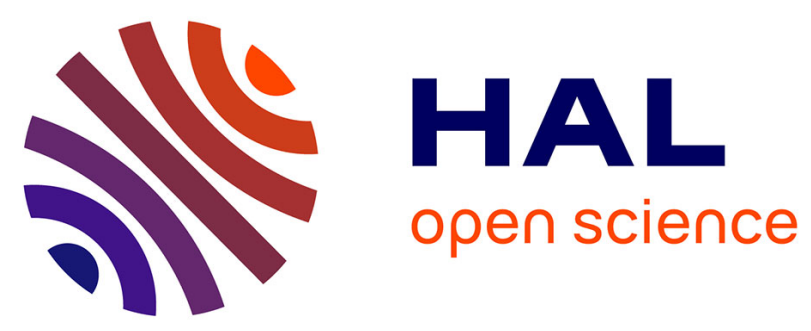

\title{
Visions et visées des formes sentencieuses dans la littérature médiévale espagnole du XIVe siècle (El Conde Lucanor et Libro de Buen Amor)
}

Alexandra Oddo

\section{- To cite this version:}

Alexandra Oddo. Visions et visées des formes sentencieuses dans la littérature médiévale espagnole du XIVe siècle (El Conde Lucanor et Libro de Buen Amor). Cahiers d'Etudes Hispaniques Médiévales, 2009, 32 (1), pp.229 - 244. 10.3406/cehm.2009.2075 . hal-01914117

\section{HAL Id: hal-01914117 \\ https://hal.parisnanterre.fr/hal-01914117}

Submitted on 6 Nov 2018

HAL is a multi-disciplinary open access archive for the deposit and dissemination of scientific research documents, whether they are published or not. The documents may come from teaching and research institutions in France or abroad, or from public or private research centers.
L'archive ouverte pluridisciplinaire HAL, est destinée au dépôt et à la diffusion de documents scientifiques de niveau recherche, publiés ou non, émanant des établissements d'enseignement et de recherche français ou étrangers, des laboratoires publics ou privés.

\section{다(1)(2)}

Distributed under a Creative Commons Attribution - ShareAlikel 4.0 International 


\title{
Visions et visées des formes sentencieuses dans la littérature médiévale espagnole du XIV ${ }^{e}$ siècle (El Conde Lucanor et Libro de Buen Amor)
}

\author{
Alexandra ODDO \\ Université Paris X - Nanterre
}

\begin{abstract}
RÉSUMÉ
Parmi les vecteurs de transmission du savoir dans la littérature du Moyen Âge, les formes sentencieuses occupent une place prépondérante, notamment dans les deux chefs-d'œuvre du XIV ${ }^{\mathrm{e}}$ siècle, le Libro de Buen Amor et El conde Lucanor. L'examen de leur présence, mais aussi l'analyse de leur dénomination, de leur positionnement et de leur référencement laissent pourtant entrevoir que la vision de l'univers proverbial des deux auteurs diffère sensiblement. Tout comme le rôle qui est alors dévolu aux énoncés sentencieux, qui remplissent dans chaque livre une mission bien particulière en accord avec les enjeux qui caractérisent les deux œuvres.
\end{abstract}

\section{RESUMEN}

Como los exempla, las formas sentenciosas se insertan en la literatura sapiencial de la Edad Media y forman parte de las estrategias argumentativas de las dos grandes obras maestras del siglo XIV, el Libro de Buen Amor y El conde Lucanor. El análisis de su presencia, así como el estudio de su denominación, de su posición textual y del sistema de referencias al que recurren ambos autores indica sin embargo que su visión en lo que se refiere a sentencias ofrece divergencias notables. De hecho, estos enunciados sentenciosos aparecen supeditados al objetivo didáctico desarrollado en cada obra, lo que explica su peculiar utilización por parte de los dos autores.

Grand nombre des chefs-d'œuvre de la littérature médiévale espagnole témoignent de l'évolution culturelle et sociétale d'une communauté qui accorde désormais au savoir une place prépondérante. À travers la littérature sapientielle, un nouvel art d'enseigner se met en place qui se propose d'apprendre aux hommes à se préserver des périls de ce monde. Ce courant littéraire, éminemment didactique, trouve tout naturellement 
dans les formes sentencieuses et dans les contes son moyen d'expression privilégié. Ces deux vecteurs du savoir sont alors intégrés à des narrations plus complexes qui permettent souvent, en dernière instance, d'en déchiffrer le sens.

Des conseils, des règles de conduite, pour une société, et plus particulièrement pour ses deux strates dominantes, les clercs et les chevaliers, qui ont une valeur universelle. Pour autant, impossible de confondre ces œuvres avec les recueils sermonnaires qui les ont précédées: cette nouvelle transmission du savoir doit être amène, facilitée par une trame narrative profondément attachée au concret et à l'expérience.

Ce courant est indissociable de la littérature espagnole au Moyen Âge. Les écrits se succèdent dès le XII ${ }^{e}$ siècle, notamment par le biais de traductions inspirées de collections orientales: Calila e Dimna ${ }^{1}$, Sendebar ${ }^{2}$, Barlaam et Fosafat ${ }^{3}$. Quant aux productions péninsulaires, elles s'inscrivent aussi dans cette volonté didactique et puisent abondamment dans diverses sources, orientale, latine, ésopique.

La critique distingue d'une façon unanime deux de ces œuvres: Libro del conde Lucanor et Libro de Buen Amor car elles constituent en quelque sorte l'aboutissement de ce courant au Moyen Âge, par leur caractère novateur dans le maniement des différentes formes brèves qui les composent. Elles ont en effet en commun d'entremêler les exempla et les formes sentencieuses dans l'élaboration de leur schéma argumentatif et de les soumettre aux objectifs didactiques affichés par les deux auteurs. Pourtant, malgré cette affinité, l'exégèse des deux textes révèle de nombreuses divergences dans l'instrumentalisation de ces vecteurs du savoir, notamment en ce qui concerne les énoncés parémiques.

Nous nous attacherons ici à analyser l'utilisation qui est faite de ces formes sentencieuses dans les deux ouvres, en les étudiant dans le contexte de la stratégie argumentative dans laquelle elles s'intègrent, afin de déterminer dans quelle mesure la visée et la vision des deux auteurs diffèrent sur cette question.

\section{Formes sentencieuses et contexte}

Pour développer leur argumentation, les auteurs ont recours au Moyen Âge aux formes brèves, dont la typologie a été minutieusement étudiée

1. José Manuel Cacho Blecua, María Jesús Lacarra, Calila y Dimna, Madrid: Castalia, 1988.

2. María Jesús LACARRA (éd.), Sendebar, Madrid: Cátedra, 1989.

3. Severiano Carnero Burgos, Barlan y Josafat, Madrid: Université Complutense, 1990. 
par Marta Haro Cortés ${ }^{4}$. Exempla, fables, allégories, similitudo, descriptio et formes gnomiques constituent ainsi le fonds commun, traditionnel ou rénové, qu'utilisent les écrivains qui s'inscrivent dans le courant de la littérature sapientielle.

Il s'agit pourtant d'un savoir en construction, des premiers pas de l'écriture en langue romane, ce qui rend malaisé le travail sur les sources et sur la définition même des concepts qui y sont manipulés. De fait, la parémiologie au Moyen Âge doit être étudiée dans un cadre assez large où prime l'idée de l'argument d'autorité, de l'affirmation à caractère universel.

La définition de Marta Haro Cortés sous-tend cette difficulté taxinomique et englobe de fait sous le terme de sentencia toutes les formes parémiques qui peuplent ces œuvres:

Considero sentencia o dicho una forma comunicativa no narrativa y normalmente breve que encierra en su seno una lección universal ya explícita o implícitamente (me refiero en este caso a que adopta una forma metafórica o retórica) referida prioritariamente al ámbito del comportamiento tanto ético-moral como en algunos casos social. También me parece viable la definición de sentencias como un nudo específico de comunicación con una estructura analógica.

C'est à partir de ce cadre, fort large, qu'on peut dire que les énoncés sentencieux sont extrêmement fréquents dans les deux œuvres et constituent, au même titre que l'exemplum, une de leurs caractéristiques majeures. Répartis d'une façon homogène dans le Libro de Buen Amor, ils révèlent chez l'auteur une véritable volonté d'utiliser ce type d'instrument dans la construction de son développement.

La materia paremiológica en el Libro de Buen Amor se reparte entre paremias y sentencias. Las paremias son casi todas refranes, y alguna frase proverbial, pero no deja de tener su presencia, aunque pequeña, proverbios clásicos y latinos medievales. El número conjunto de refranes y frases proverbiales puede pasar del centenar (parte del material refranístico está por documentar y, sin duda, nunca se conseguirá hacerlo del todo). Las sentencias son incalculables ${ }^{6}$.

Une autre impression se dégage de la lecture du Libro del Conde Lucanor. Il est question en effet d'une division entre les contes et les énoncés parémiques, dont fait état le prologue: une première partie étant consacrée aux exempla et les parties suivantes aux formes sentencieuses. Cette lecture serait néanmoins superficielle puisque de nombreuses formes sentencieuses agrémentent le premier livre, comme le précise sa quatrième partie:

4. Marta HaRO CORTÉS, Los compendios de castigos del siglo XIII: técnicas narrativas y contenido ético, Université de Valence, Cuadernos de filología, annexe XIV, 1995.

5. Ibid., p 115.

6. María Pilar CuARTero SANCHO, «La paremiología en el Libro de Buen Amor», http:// cvc.cervantes.es/obref/arcipreste_hita/cuartero.htm, 23 pages. 
[Cuarta parte del libro del conde lucanor et de Patronio. Razonamiento de Patronio al Conde Lucanor] Et assí, con los exiemplos et con los proverbios, hevos puesto en este libro dozientos entre proverbios y exiemplos, et más: ca en los çincuenta exiemplos primeros, en contando el exiemplo, fallaredes en muchos lugares algunos proverbios tan buenos et tan provechosos commo en las otras partes deste libro en que son todos proverbios?

Or, cette forte présence de l'univers proverbial dans les deux œuvres ne peut pas être analysée en dehors du contexte qui l'accueille. Dans le Libro de Buen Amor, nous nous trouvons face à une structure où s'enchaînent les épisodes amoureux et les exempla. Les formes sentencieuses jalonnent ${ }^{8}$ l'œuvre $^{\prime}$ de l'archiprêtre de Hita. Elles peuvent apparaître seules au sein d'un développement, dans des positions textuelles diverses (initiale, interne ou finale):

Ca, puesto que su signo sea de tal natura como es este mío, dize una escriptura que «buen esfuerzo vençe a la mala ventura", e a toda pera dura grand tiempo la madura

Non perderé yo a Dios nin al su paraíso por pecado del mundo, que es sonbra de aliso; non soy yo tan sin seso, [que] si algo he priso, quien toma dar deve ${ }^{9}$, dízelo sabio enviso

En la cama muy loca, en la casa muy cuerda ${ }^{10}$, Non olvides tal dueña, mas d'ella te acuerda;

Esto que te castigo con Ovidio concuerda, E para aquésta, cata la fina avancuerda.

ou alors être associées à un exemplum, ce qui se réalise très fréquemment dans le texte ${ }^{11}$. Le premier conte de l'œuvre «La dispute qui s'éleva entre les Grecs et les Romains » en constitue un exemple intéressant, puisqu'un proverbe va lui servir de conclusion:

Non ha mala palabra si non es a mal tenida.

7. Don Juan Manuel, El Conde Lucanor, José Manuel Cacho Blecua (éd.), Madrid: Castalia, 2003, p. 295.

8. Un relevé non exhaustif des formes sentencieuses présentes dans le Libro de Buen Amor montre que ceux-ci sont exploités d'une façon régulière tout au long de l'œuvre. Voir Bernard DARBORD, Alexandra ODDO BONNET, «Les caractéristiques du proverbe médiéval. Libro de Buen Amor», in: Mélanges en hommage à Madeleine et Arcadio Pardo, Université Paris X-Nanterre, Centre de recherches ibériques et ibéro-américaines, 2008.

9. «Quien toma dar deve»: Gregorio Doval, Refranero temático español, Madreid: Ediciones del Prado, 1997.

10. «A la noche putas, y a la mañana comadres»: Gonzalo CORREAS, Vocabulario de refranes, Louis Сомвет (éd.), Université de Bordaux, Institut d'études ibériques et ibéro-américaines, 1967.

11. Sur les relations qu'entretiennent les proverbes et les exempla dans le Libro de Buen Amor: Alexandra ODDo BONNET, «L'art d'enseigner dans le Libro de Buen Amor. Analyse d'une relation complémentaire proverbe/exemplum», actes du colloque «Les formes brèves », EA 369, Université Paris Ouest La défense, mars 2008. 
L'apparition des énoncés parémiques semble beaucoup plus conditionnée dans le Libro del Conde Lucanor. El libro de los exemplos ${ }^{12}$ possède une structure unique qui se répète en associant toujours les mêmes ingrédients: une problématique entraîne l'insertion d'un exemplum dans le dialogue, automatiquement suivi d'une conclusion et des viessos, là où se concentrent d'ailleurs les formes sentencieuses ${ }^{13}$ :

Exemplum 2: De lo que contesçió a un omne bueno con su fijo.

Conclusion de Patronio: en lo que oviéredes de fazer, et lo fallarédes que es bien et vuestra pro; consejóros yo que nunca lo dexedes de fazer por reçelo de lo que las gentes podrían dello dezir. Forme recueillie par Hernán Núñez, Seniloquium: Que quiera que digan las gentes, a ti mismo para mientes.

Exemplum 7: De lo que contesçió a una muger quel dizién doña Truhana.

Conclusion de Patronio: cred et cuydat sienpre todas cosas tales que sean aguisadas et non fuzas dubdosas et vanas.

Forme recueillie par Correas: No se deve dexar lo zierto por lo dudoso e inzierto.

A eso me atengo, ke es lo zierto.

Viessos: A las cosas çiertas vos comendat / et las fuyzas vanas dexat

Exemplum 20: De lo que contesçió a un rey con un omne quel dixo quel faría alquimia Viessos: Non aventuredes mucho la tu riqueza, / por conseio de[l] que a grand pobreza Forme recueillie par Correas: No tomes konsexo de tu rrikeza kon el onbre ke está en pobreza

Dans les parties suivantes, consacrées aux énoncés sentencieux, ceux-ci apparaissent au gré du dialogue et sont indépendants dans leur fonctionnement:

Et pues el otro es acabado, este libro comiença assí:

- En las cosas que ha muchas sentençias, non se puede dar regla general.

- El más complido de los omnes es el que cognosce la verdat et la guarda.

- De mal seso es el dexa et pierde lo que dura et non ha preçio, por lo que non puede aver término a la su poca durada ${ }^{14}$.

Ces répliques «sentencieuses» ont été analysées par Knust au début du $\mathrm{XX}^{\mathrm{e}}$ siècle dans son édition du Libro del Conde Lucanor ${ }^{15}$. Pour autant, elles posent toujours problème à la critique : si celle-ci conçoit cette partie

12. La critique a longtemps été divisée quant au nombre d'articulations, deux ou trois parties, avec une constante concernant la spécificité du premier livre (Libro de los exemplos) face aux autres (Libro de los proverbios). Voir DON JUAN MANUEL, El Conde Lucanor, Guillermo SERÉs (éd.), Barcelone: Biblioteca Clásica, Crítica, 1994, p. LXIV-LXV.

13. Bernard DARBORD, «La parole proverbiale de don Juan Manuel», in: Hommage à JeanClaude Anscombre, à paraître.

14. Don Juan Manuel, El Conde Lucanor, J. M. Cacho Blecua (éd.), op. cit., p. 279.

15. Juan MANueL, El libro de los enxiemplos del conde Lucanor et de Patronio, Hermann KNUST (éd.), Leipzig: Dr Seele Co., 1900. 
du livre comme émanant du genre sentencieux, les rapprochements avec les proverbes attestés n'en restent pas moins malaisés, voire impossibles dans certains cas:

Es cierto que el número de sentencias conservado no se corresponde al que anuncia Patronio en las introducciones de cada una de estas partes, lo que ha motivado correcciones de orden textual. En todo caso, parece claro que esta organización de ideas poseía una unidad propia, vinculada a la tradición genérica de la que procede esta forma especifica de acercarse al saber ${ }^{16}$.

Il s'avère en effet que la très grande majorité des énoncés sentencieux qui apparaissent dans l'œuvre ne sont pas attestés comme tels ${ }^{17}$. Dans l'édition de José Manuel Cacho Blecua, il est fait état de cinq proverbes attestés, qui ne peuvent en aucun cas refléter réellement le travail accompli par l'auteur pour couler bon nombre de ses assertions dans le moule de la formulation parémique.

Les données quantitatives sont donc plus difficiles à produire dans le cadre de l'œuvre de don Juan Manuel. D'autant que souvent, le proverbe est reconnu comme tel dans la mesure où il est introduit dans le texte par le biais d'un élément d'insertion visant à l'identifier. Dans le Libro de Buen Amor, les modalités d'insertion sont variées et servent l'objectif assigné aux formulations sentencieuses en réitérant leur validité. Ainsi, se côtoient des dénominations variées ${ }^{18}$ : la fabla, la fablilla, el proverbio, los antiguos retráheres souvent associées à l'idée de véracité ou de sagesse : dize verdat la fabla; verdat es lo que dizen los antiguos retráheres, dizelo sabio enviso.

Dans le Libro del Conde Lucanor, le système de référencement est tout à fait différent: les dénominations, essentiellement palabra, sentencia, proverbio et vierbo, apparaissent désolidarisées de leur expression concrète dans le texte. Autrement dit, le cadre est posé d'une narration où se produisent des énoncés parémiques, sans que toutefois ceux-ci soient identifiés comme tels dans le récit ${ }^{19}$.

Si cet état de fait peut s'expliquer en partie par la nature même des séquences qui intègrent les formulations sentencieuses, les viessos étant des créations poétiques qui visent l'équilibre métrique, nous ne pouvons que

16. Don Juan Manuel, El Conde Lucanor, J. M. Cacho Blecua (éd.), op. cit., p. XXXVIII.

17. L'attestation des formes se réalise par le recoupement avec d'autres œuvres. Dans le cas du Libro del Conde Lucanor, les correspondances ne sont pas relevées dans Eleanor S. O'KANE, Refranesy frases proverbiales españolas de la Edad, Madrid: Anejos del boletín de la Real Academia, II, 1959.

18. À ce sujet, voir Bernard DARBord, Luz VAlle VIDELA, «Réflexion sur la technique de l'exemplum dans le Libro de buen amor: fazañas, fablillas, parlillas, proverbios non mintrosos», in: El libro de buen amor de Juan Ruiz, archiprêtre de Hita, Carlos HEUscH (éd.), Paris: Ellipses, 2005, p. 99-113.

19. Quelques exceptions doivent être soulignées dans ce système: «Non quiso fazer viessos de nuebo, sinon que puso y una palabra que dizen las viejas en Castiella. Et la palabra dize así: Quien bien siede non se lieve», p. 78; «commo dezía el viebo antiguo: murió el onbre et murió el su nombre», p. 116; in: Don Juan Manuel, El Conde Lucanor, J. M. Cacho Blecua (éd.), op. cit. 
constater à de nombreuses reprises les ressemblances avec des formulations sentencieuses attestées dans les recueils ${ }^{20}$ :

Exemplum 46: De lo que contesçió a un philósopho que por ocasión entró en una calle do moravan malas mugeres.

Viessos: Faz siempre bien et guárdate de sospecha, / et siempre será la tu fama derecha.

Forme recueillie par Correas: En ora buena naze kien buena fama kobra i por tenerla haze o $i$ por guardarla haze.

Exemplum 42: De lo que contesçió a una falsa veguina.

Viessos: Para mientes a las obras et non a la semejança, / si cobdiçiares ser guardado de aver mala andança.

Forme recueillie par Correas: Por las obras, no por el vestido, el hipócrita es conoscido. Forme recueillie par Hernán Núñez: De juizios no me curo, que mis obras me hazen seguro.

Ces analogies sont aussi fréquentes lors des conclusions de Patronio:

Exemplum 2: De lo que contesçió a un omne bueno con su fijo

[...] consejóvos yo que nunca lo dexedes de fazer por reçelo de lo que las gentes podrían dello dezir.

Forme recueillie par Hernán Núñez, Seniloquium: Que quiera que digan las gentes, a ti mismo para mientes.

Exemplum 23: De lo que fazen las formigas para se mantener

[...] ca çierto sed que por grant aver que sea, onde sacan cada día et non ponen y nada, que non puede durar mucho.

Forme recueillie par Correas: Donde ai saka y nunka pon, presto se akaba el bolsón.

L'explication de ce manque de référencement, qui peut manifester une véritable volonté de la part de l'auteur, doit probablement être mise sur le compte du contexte littéraire qui conditionne l'apparition de ces formulations sentencieuses. En effet, contrairement au Libro de Buen Amor, où le narrateur-protagoniste prend en charge le récit, nous sommes, dans le cas du Libro del Conde Lucanor, face à un récit-cadre dans lequel alternent les figures de l'apprenant et du conseiller. Ce dernier symbolise la fonction primordiale du livre, il est l'incarnation de la sagesse, et c'est grâce à lui que va se mettre en place le savoir pratique, actif, qui consiste à être en position de faire le bon choix. Inscrit dans le deuxième genre rhétorique, le genre délibératif suppose que le conseiller a choisi au préalable la démarche à suivre et il en découle que la sagesse peut directement émaner de lui.

La sagesse, dans ce cadre, peut fonctionner en dehors de ses sources, et consolider, au même titre que l'exemplum, le développement de l'argumentation.

20. «Su segunda misión es la composición de unos viessos, en los que se recoja la intención del ejemplo. Con ellos el mensaje didáctico de Patronio alcanza su máximo nivel de generalización y se refuerza la evidente relación con el contenido de las tres partes siguientes, dedicadas sólo a proverbios », in: María Jesús LACARRA, Cuentos de la Edad Media, Madrid: Castalia, 1989, p. 72. 


\section{Au sujet des stratégies d'argumentation}

Pour analyser les schémas argumentatifs développés par les deux auteurs, nous devons tenir compte des spécificités de chaque œuvre. Autrement dit, il faut mettre en relation les différentes formes brèves qui se côtoient dans le texte pour déterminer comment fonctionnent ces énoncés parémiques au regard de la stratégie textuelle mise en place, et surtout, car il s'agit bien là de l'objet de cette étude, pour définir dans quelle mesure un instrument commun peut être envisagé de deux façons différentes.

L'analyse de la relation qui existe entre l'exemplum et les formes sentencieuses dans les deux textes permet en effet de mieux définir leurs rôles respectifs dans l'argumentation. Dans cette optique, une analyse de leur positionnement peut se révéler féconde et apporter des réponses fonctionnelles.

Dans le Libro de Buen Amor, nous nous trouvons face à une disposition qui, de prime abord, ne semble pas correspondre à des critères systématiques. Disséminées tout au long de l'œuvre, les formes sentencieuses sont parfois rattachées à des exempla, le nombre de proverbes associés pouvant aller jusqu'à trois.

On constate une forte concentration de formes sentencieuses accolées aux exempla au début du texte, alors que lors du développement, cette association semble être due au hasard. La disposition, à proprement parler, des formes sentencieuses, est variable suivant les contes. Les cas où le proverbe apparaît comme support du conte sont moins nombreux ${ }^{21}$ et se manifestent aussi au début de l'œuvre.

Par la suite, le positionnement adopté devient plus régulier, les formes sentencieuses se situant essentiellement après les exempla. Dans cette configuration elles servent l'argumentation en proposant une conclusion ou une illustration (souvent ornementale) au concept développé.

Cette modularité est remarquable puisque le positionnement détermine l'affectation dans le processus qui consiste à convaincre le récepteur du message. Dans le cas du Libro de Buen Amor, les proverbes et les sentences peuvent exercer diverses fonctions rhétoriques en ce sens où ils peuvent être le point de départ d'une argumentation ou sa conclusion.

21. Positionnement des proverbes: après le conte dans «La dispute qui s'éleva entre les grecs et les romains» et «L'horoscope du roi Alcaraz». D’autres exempla se retrouvent encadrés par les formes sentencieuses: «Le partage du lion» s'ouvre et se ferme grâce à l'élément proverbial. Quant à «La montagne qui accouche» et «Le chien et le voleur», ils ont dans leurs sillages deux proverbes pour les introduire et un troisième qui fait office de conclusion à l'argumentation,. Voir A. ODdo Bonnet, «L'art d'enseigner dans le Libro de Buen Amor. Analyse d'une relation complémentaire proverbe/exemplum.», art. cité. 
On arrive vite aux réflexions d'ordre stylistique, par exemple, à quel endroit faut-il introduire proverbes et sentences pour convaincre ou pour agrémenter?

Dans les deux fonctions, d'ornatus et d'auctoritas, les possibilités d'exploiter un noyau d'idées générales sont en principe les mêmes. La place dans le discours contribue toutefois à fixer le statut de preuve ou d'ornement ${ }^{22}$.

La fréquente postposition discursive de l'élément proverbial fait donc apparaître une volonté de la part de l'auteur de s'en remettre à l'argument d'autorité, garant de l'adhésion du récepteur, d'autant qu'il s'agit prioritairement de proverbes attestés dans l'œuvre de l'archiprêtre de $\mathrm{Hita}^{23}$, comme le montrent ces quelques exemples.

Le partage du Lion

El cuerdo y la cuerda en mal ageno castiga.

L'horoscope du roi Alcaraz

Aunque omne non goste la pera del peral, en estar a la sonbra es plazer comunal.

Quien en el arenal siembra non trilla pegujares.

Quien toma dar debe.

Vulpes, le renard qui fait le mort

Lo que emendar non se puede non presta arrepentir.

Virgile l'enchanteur

Por malas vezindades, se pierden eredades.

Des arguments d'autorité, donc, qui recouvrent dans le cas du Libro de Buen Amor une autre fonction spécifique au regard des exempla qu'ils accompagnent. En effet, une analyse approfondie des relations qui unissent les contes aux formes sentencieuses permet de constater que ces dernières obéissent à un autre critère fonctionnel dans l'argumentation. Nous avons évoqué précédemment la distribution des sentences et des proverbes par rapport aux exempla et constaté que certains contes se présentaient en l'absence d'énoncés parémiques les accompagnant. Or, ce qui pourrait relever de l'aléatoire ne l'est en fait pas du tout: les formes sentencieuses offrent, dès lors qu'elles sont présentes, des clés de lecture pour l'exemplum.

Ainsi, «Le partage du lion», «La montagne qui accouche», «Le chien et le voleur», «Le loup et la grue», «Le paon et la corneille», «Le lion vieilli» parmi d'autres, nous permettent de vérifier cette fonction. Ces contes offrent tous la particularité de développer plusieurs idées centrales ou plusieurs morales. Or, cette multiplication des leçons ou des axes de lecture

22. Elizabeth SchUlZE-BuSACKER, «La place du proverbe dans la mentalité médiévale», Paremia, 6, 1997, p. 567.

23. «Les proverbes cités au discours tirent leur autorité du fait d'être universellement connus, tandis que les sentences expriment «une connaissance généralement admise, posée comme universelle», ibid. p. 566. 
déclenche et conditionne l'apparition d'une forme sentencieuse, en général après l'exemplum.

À l'inverse, les exempla qui fonctionnent de façon autonome et dont la lecture est univoque n'auront pas besoin d'avoir dans leur sillage une forme sentencieuse dont la fonction serait de lever les ambiguités ${ }^{24}$.

Les énoncés parémiques du Libro de Buen Amor possèdent de ce fait une double fonctionnalité: dans un premier temps, ils orientent la lecture et deviennent dès lors le garant d'une réception cohérente de l'argumentation. Puis, dans leur fonction rhétorique et du fait de leur nature même d'argument d'autorité, ils sont les vecteurs privilégiés de la transmission du savoir et offrent des conclusions universelles et reconnues qui facilitent l'adhésion du récepteur aux idées développées dans l'argumentation.

En ce qui concerne le texte de don Juan Manuel, nous allons voir que les enjeux et, par conséquent, la vision du rôle que doivent jouer les formes sentencieuses dans l'argumentation est tout à fait différente. Il ressort de la lecture des prologues que les formes brèves sont, suivant leur nature, plus ou moins accessibles au public, même si elles possèdent la caractéristique commune d'être salutaires et profitables. Il est à ce sujet utile de comparer le prologue:

Por ende yo, don Fohan, fijo del infante don Manuel, adelantado mayor de la frontera et del regno de Murçia, fiz este libro de las más apuestas palabras que yo pude, et entre las palabras entremeti algunos exiemplos de que se podrían aprovechar los que los oyeren ${ }^{25}$

à la Escusación de Patronio al Conde Lucanor, qui introduit la troisième partie du livre:

- Señor conde Lucanor - dixo Patronio-, después que el otro libro fue acabado, porque entendí que lo queriades vós, començé a fablar en este libro más abreviado et más oscuro que en l'otro. Et commo quier que en esto que vos he dicho en este libro ay menos palabras que en el otro, sabet que non es menos el aprovechamiento et el entendimiento deste que del otro, antes es muy mayor para quien lo estudiare et lo entendiere ${ }^{26}$.

C'est à partir de ce principe que se comprend la division du livre en parties comprenant soit des exempla, soit des sentences, puisque ces dernières se caractérisent par une trop grande obscurité ${ }^{27}$, qui les met hors

24. Sur l'ambiguïté de certains contes et la multiplication des lectures, voir la typologie proposée par Félix LECOY, Recherches sur le Libro de buen amor de fuan Ruiz, archiprêtre de Hita, Paris : Droz, 1938; et l'article de Ian MichaEL, «The function of the popular tale in the Libro de buen amor», Libro de buen amor's Studies, G. B. Gybbon-Monypenny (éd.), Londres: Tamesis Books, 1970, p. 177-218.

25. Don Juan Manuel, El Conde Lucanor, J. M. Cacho Blecua (éd.), op. cit., p. 50.

26. Ibid, p. 288.

27. L'opacité des proverbes serait liée à leur généralité et à leur brièveté, à la difficulté d'appréhender des concepts sans l'aide d'un exemplum fourni au préalable. À ce sujet voir M. J. LACARRA, op. cit., p. 74. 
de portée de certains lecteurs. Mais cette dissociation n'est qu'apparente dans le premier livre, puisque l'argumentation est en fait très structurée, et que la formulation proverbiale ${ }^{28} \mathrm{y}$ joue un rôle prépondérant.

En effet, l'argumentation aura toujours comme point d'ancrage l'exemplum dans la première partie du livre. Pour autant, il ne fonctionne jamais indépendamment du contexte posé par le récit-cadre qui consolide l'argumentation en enchaînant les différents instruments à caractère didactique dans le sillage de l'exemplum. Il s'agit d'un schéma classique au Moyen Âge, qui préfère les raisonnements analogiques aux techniques inductives. La transmission du savoir est par ce biais facilitée et la formulation sentencieuse retrouve, en position conclusive, son statut de preuve:

Lo didáctico exige una progresiva generalización de la enseñanza a través de un proceso de abstracción gradual: situación del ejemplo, situación del conde y situación del lector. Universalizar la moraleja es en definitiva, la función más especifica del marco. [...] Al fin y al cabo, es el consejero quien fija el sentido último de lo narrado, quien da la interpretación válida del cuento y quien lo orienta moral o doctrinalmente ${ }^{29}$.

D’ailleurs, les autres parties du livre ne sont que le reflet de ce schéma didactique: les mêmes thèmes y sont développés, sans avoir recours, puisqu'il s'agit d'une acquisition graduée du savoir, à l'analogie métaphorique ou métonymique que suppose l'exemplum. Points culminants de l'argumentation, les formes sentencieuses peuvent alors exister indépendamment des autres formes brèves.

\section{Au sujet des enjeux}

Il ressort de cette analyse que l'emploi des formes sentencieuses ne répond pas aux mêmes objectifs dans ces deux œuvres. Si Juan Ruiz les utilise pour lever des ambiguités et pour asseoir la validité de ses arguments, le système graduel développé par don Juan Manuel semble davantage se fonder sur une acquisition progressive du savoir où priment les notions d'efficacité et de style.

Ce rapport au savoir est présenté par les auteurs dans le prologue et de fait, leur attitude diffère déjà dans la relation qu'ils établissent avec leurs récepteurs. Le prologue est au Moyen Âge une étape décisive de la création litté-

28. Les viessos et les conclusions de Patronio adoptent fréquemment toutes les caractéristiques de l'énoncé proverbial. Les conclusions de Bernard Darbord au sujet des viessos de don Juan Manuel sont probantes («La parole proverbiale de don Juan Manuel», in: Hommage à Jean-Claude Anscombre, à paraître). Au sujet des conclusions de Patronio, voir Alexandra ODDO BONNET, «Les traces de l'univers parémiologique dans El conde Lucanor de don Juan Manuel», colloque de linguistique ibérique et ibéro-américaine, Rennes, septembre 2008, à paraître.

29. Don Juan Manuel, El Conde Lucanor, J. M. Cacho Blecua (éd.), op. cit., p. LVIII. 
raire. Une véritable tradition qui vise à faciliter l'accès au contenu du texte, à éveiller l'intérêt du lecteur mais aussi à orienter sa lecture en la guidant.

La lecture de ces deux prologues est porteuse de sens dans la perspective de notre analyse et montre en effet que le processus d'acquisition du savoir par le récepteur est en quelque sorte inversé dans les deux œuvres. Juan Ruiz enchaîne les épisodes amoureux, contre exemples du Buen Amor, celui-là même qui doit amener le lecteur au salut. Il classe ses lecteurs en fonction de leur capacité de compréhension et du profit qu'ils pourront tirer de leur lecture. De son schéma se dégagent trois types de lecteurs. Celui qui, sollicitant son intellect, deviendra le destinataire privilégié de l'œuvre, distinguera le bien du mal et travaillera, grâce à l'application de ses préceptes, pour le salut de son âme. Celui qui, sollicitant sa mémoire, trouvera dans le livre les exemples a contrario qu'il ne doit pas suivre, même si ses capacités de compréhension sont moindres. Puis, finalement, le pécheur, qui suivra sans discernement la pratique du fol amour sans penser à son salut. La dimension didactique de l'œuvre est assujettie à la perspective religieuse et fortement enracinée dans la pensée chrétienne.

L'œuvre de don Juan Manuel, très fortement inscrite elle aussi dans cette mentalité - il est question du salut de l'âme dès les premières lignes du prologue - est profondément en accord avec le statut social de son auteur et par conséquent très ancrée dans le concret, dans le savoir pratique ${ }^{30}$. Quant à ses destinataires, ils ne font pas réellement l'objet d'un classement: «et porque $[a]$ muchos omnes las cosas sotiles non les caben en los entendimientos, porque non las entienden bien [...]». L'élément primordial reste la transmission du savoir, un savoir sans ambiguité mis à la portée de tous.

Ces deux différentes approches du savoir vont par voie de conséquence attribuer un rôle particulier aux formes sentencieuses, en fonction du contexte d'énonciation dans lequel elles s'insèrent. Chez Juan Ruiz, la trame narrative et parfois même l'exemplum peuvent fonctionner comme des contre exemples. Le savoir est caché - c'est souvent le cas dans la littérature sapientielle - et exige de la part du destinataire une démarche intellectuelle de recherche du sens. Dans ce parcours, les énoncés sentencieux qui jalonnent le texte vont lui permettre de lever les ambiguités inhérentes à la construction du texte.

Don Juan Manuel, quant à lui, mise sur une autre approche. La construction se fait au profit d'un message qui ne doit souffrir aucune altération. Dans ce contexte, les formulations sentencieuses doivent être adaptées,

30. «Nótese que no se trata sólo de salvar las almas, sino también de "aumentar onras", "faziendas" y "estados", lo que es harto significativo. Don juan Manuel, como todos los moralistas medievales, estaba muy preocupado con la salvación del alma (la última parte del libro girará alrededor de este problema) pero luchó muchas veces por cuestiones de "honra" y de "hacienda" ", in: DON JuAN MANUEL, El Conde Lucanor, J. M. GaChO Blecua (éd.), op. cit., p. 28. 
voire créées - même si leur forme et leur contenu relèvent toujours de la parémiologie - et sont de fait toujours subordonnées à la leçon que renferme l'exemplum.

Il faut toujours avoir à l'esprit que la littérature médiévale possède des règles qui lui sont propres, notamment en matière d'utilisation des sources. La littérature est très liée à l'oralité et la tradition très présente dans les textes. Elle se traduit par l'utilisation de topoi, de références facilement identifiables et qui de fait créent un lien entre l'auteur et son public. Dans le cas du Libro de Buen Amor, l'auteur fait appel aux textes des Pères de l'Église, à la sagesse populaire, aux écrits latins: nul doute qu'il ne cache pas ses sources. Les formes sentencieuses sont soumises aux contraintes des enjeux didactiques, leur vocation est de ce fait plus explicative qu'illustrative au sein de l'argumentation développée par l'auteur. Essentielles dans l'économie de l'œuvre, elles permettent de guider la compréhension du lecteur et deviennent, de ce fait, le lieu privilégié de la démarche didactique dans le Libro de Buen Amor.

L'analyse de ces formes dans l'œuvre de don Juan Manuel doit passer par l'adoption d'une autre perspective. Dans El Conde Lucanor, les énoncés sentencieux apparaissent comme dilués dans le texte, entièrement solidaires de leur contexte. On y constate une véritable volonté d'innovation et de création dans ce domaine qui laisse peu de place à l'utilisation des formes attestées. L'objectif semble donc être dans son cas de renouveler le matériau des proverbes traditionnels grâce à des reformulations souvent mimétiques fondées sur un rapport de proximité sémantique et syntaxique. La présence d'énoncés parémiques y est bien flagrante, mais l'absence de référencement et d'attestation les transforme en produits de l'auteur, et non plus de la sagesse populaire.

Ce faisant, l'auteur parvient à créer une véritable harmonie entre les différents vecteurs du savoir présents dans l'œuvre. Aucune ambiguïté ne naît de la superposition des formes brèves et tous les éléments concourent dans le texte à transmettre un message univoque: «entendí que sería más ligero de entender [...] fiz las razones et exiemplos que en el libro se contienen assaz llanas et declaradas $»^{31}$. D'autant que les formes sentencieuses à proprement parler, et les parties qui les contiennent font l'objet d'un jugement critique: elles sont jugées obscures. Ce qui pourrait expliquer cette préférence de l'auteur pour un matériau renouvelé.

D'autre part, la démarche de l'auteur doit aussi être reliée à sa propre personnalité et à sa volonté de créer une œuvre originale. El conde Lucanor occupe une place tout à fait particulière dans la littérature du Moyen Âge, qui a été maintes fois soulignée par la critique: 
La obra literaria de la Edad Media es esencialmente una obra de compilación de materiales ya existentes que se organizan en formas diversas y se pasan a la posteridad. De alli que el estudio de la fuentes literarias sea tan importante. Respecto a don Fuan Manuel se ha hecho notar que no menciona sus fuentes sino en muy pocos casos, al contrario de otros escritores de esa época. Yo me atrevería a sugerir que el autor tiene una gran conciencia de la obra que lleva a cabo, de su creación y de los cambios esenciales a que va sometiendo el material laborable; y así, sabiendo que difiere sustancialmente, no tiene para qué mencionar las fuentes de su inspiración ${ }^{32}$.

L'aspiration de don Juan Manuel à créer, de son propre aveu, une œuvre personnelle a certainement guidé son travail de réélaboration et de réappropriation du matériau traditionnel d'exempla et de sentences, renouveau qui contribue indéniablement à rendre cette œuvre originale et incontournable ${ }^{33}$.

L'analyse en contexte des formes sentencieuses présentes dans les deux textes permet d'avancer que Juan Ruiz et don Juan Manuel ne partagent pas la même vision de l'univers proverbial, notamment en termes de fonctionnalité dans la stratégie argumentative. Dans le Libro de Buen Amor, l'enjeu est de taille, la bonne compréhension doit permettre l'accès au salut. Les formes sentencieuses, arguments d'autorité empruntés au fonds traditionnel, sont subordonnées au message et permettent, en levant les ambiguïtés inhérentes au contenu du texte, de guider le lecteur en quête de sagesse. Enjeux et contenus qui diffèrent dans El conde Lucanor, et qui de fait vont permettre à son auteur de soumettre les formes brèves aux objectifs qu'il a assignés à son œuvre. Les sujets abordés, étroitement liés à des situations concrètes, élaborent un art de bien vivre. Dans ce contexte, la production d'énoncés sentencieux originaux, en parfaite adéquation avec les leçons développées, répond certainement au désir affiché par l'auteur d'offrir à ses lecteurs un texte accessible. Mais aussi et surtout une œuvre originale où la tradition cède sa place à la création et à la modernité.

32. Reinaldo AYERBE-CHAUX, El Conde Lucanor, materia tradicionaly originalidad creadora, Madrid: Ediciones José Porrúa Turanzas, 1975, p. XIV.

33. «Aunque don Juan Manuel aduzca con frecuencia lo que oyó contar a tal obispo, o a tal gran señor del tiempo de su padre, o a los falconeros de tal casa noble, o recuerde "fabliellas" y antiguos dichos castellanos, éstos son recursos de factura de su obra, pero en verdad su postura personal en el proceso de creación literaria da la espalda a lo que llamaríamos cultura oral, porque su objetivo es siempre la factura de un libro», in: DON JUAn Manuel, El Conde Lucanor, étude préliminaire de Germán ORduña, G. SERÉs (éd.), Barcelone: Biblioteca Clásica, Crítica, 1994, p. XXV. 


\section{Bibliographie}

\section{Sources}

ARCIPRESTE DE HitA, Libro de buen amor, edición de G. B. GybBOn-MONYPENNY, Madrid, Castalia, 1989.

Don Juan Manuel, El Conde Lucanor, edición de José Manuel Cacho Blecua, Madrid, Castalia, 2003.

\section{Sources complémentaires}

ANÓNIMO, Refranes glosados, 1541, Madrid: Real Academia Española, Corpus diacrónico del español (CORDE), http:/ /www.rae.es.

ANÓnIMO, Seniloquium, 1450, Madrid, Real Academia Española, Corpus diacrónico del español (CORDE), http://www.rae.es.

Correas Gonzalo, Vocabulario de refranes, 1627, Louis Combet (éd.), Université de Bordeaux, Institut d'études ibériques et ibéro-américaines, 1967.

De HoROzCo Sebastián, Libro de los proverbios glosados, 1570-1580, Jack Weiner (éd.), Kassel: Reichenberger, 1994.

Don Juan Manuel, El Conde Lucanor, Guillermo Serés (éd.), Barcelone: Biblioteca Clásica, Crítica, 1994.

NúÑEZ Hernán, Refranes o proverbios en romance, 1555, L. Combet, J. Sevilla, G. Conde et J. Guia (éd.), Madrid: G. Blázquez, 2001.

\section{Études}

AYERBE-CHAUX Reinaldo, El Conde Lucanor, materia tradicionaly originalidad creadora, Madrid: Ediciones José Porrúa Turanzas, 1975.

Guartero Sancho María Pilar, «La paremiología en el Libro de Buen Amor», http://cvc.cervantes.es/obref/arcipreste_hita/cuartero.htm, 23 pages.

DARBORD Bernard, ODDO BONNET Alexandra, «Les caractéristiques du proverbe médiéval. Libro de Buen Amor», in: Hommage à Madeleine et Arcadio Pardo, Université Paris X-Nanterre, Centre de recherches ibériques et ibéro-américaines, 2008.

DARBORD Bernard, VALLE VIDELA Luz, «Réflexion sur la technique de l'exemplum dans le Libro de buen amor: fazañas, fablillas, parlillas, proverbios non mintrosos», in: El libro de buen amor de Juan Ruiz, archiprêtre de Hita, Carlos Heusch (éd.), Paris, Ellipses, 2005, p. 99-113.

DARBORD Bernard, «La parole proverbiale de don Juan Manuel», in: Des topoï à la théorie des stéréotypes en passant par la polyphonie et l'argumentation dans la langue, Chambéry: Université de Savoie, 2008, p. 203-220.

Doval Gregorio, Refranero temático español, Madrid: Ediciones del Prado, 1997.

García Campos Juana, Barella Ana, Diccionario de refranes, Madrid: Espasa Calpe, 1993.

HARO CORTÉS Marta, Los compendios de castigos del siglo XIII: técnicas narrativas y contenido ético, Université de Valence, Département de philologie espagnole, 1995. 
LACARRA María Jesús, «El Libro de buen amor, ejemplario de fábulas a lo profano », in: Tipología de las formas narrativas breves románicas medievales, Juan PAREDES et Paloma Gracia (éd.), Université de Grenade, 1996, p. 237-252.

- Cuentos de la Edad Media, Madrid: Castalia, 1989.

Le Goff Jacques, Bremond Claude, SCHMitT Jean-Claude, L'exemplum, Turnhout: Brepols, 1982.

LECOY Félix, Recherches sur le Libro de buen amor de fuan Ruiz, archiprêtre de Hita, Paris: Droz, 1938.

REAl ACADEMIa EsPaÑola, Corpus diacrónico del español (CORDE), banque de données en ligne, http://www.rae.es.

SCHULZE-BUSACKER Elizabeth, «La place du proverbe dans la mentalité médiévale», Paremia, 6, 1997, p. 565-576. 